\title{
The novel IncRNA PTTG3P is downregulated and predicts poor prognosis in non-small cell lung cancer
}

Hai-Tao Huang ${ }^{1}$, Yi-Ming X ${ }^{1}$, Sheng-Guang Ding ${ }^{1}$, Xiao-Qiang Y ${ }^{1}$, Fei Wang ${ }^{1}$, Hai-Feng Wang ${ }^{2}$, Xue Tian'3 , Chong-Jun Zhong ${ }^{1}$

1Department of Thoracic and Cardiovascular Surgery, Nantong First People's Hospital, Nantong, Jiangsu Province, China

${ }^{2}$ Division of Geriatrics, Tongji Hospital, School of Medicine, Tongji University,

Shanghai, China

${ }^{3}$ Department of Respiratory Medicine, Shanghai General Hospital, Shanghai Jiaotong

University, Shanghai, China

Submitted: 28 September 2018

Accepted: 4 January 2019

Arch Med Sci 2020; 16 (4): 931-940

DOI: https://doi.org/10.5114/aoms.2020.93535

Copyright $\odot 2020$ Termedia \& Banach

\section{Abstract}

Introduction: Lung cancer is the leading cause of cancer-associated mortality worldwide. Recently, long non-coding RNAs (IncRNAs) have been studied as key regulators in some biological processes. Of note, the molecular mechanism and prognostic value of IncRNAs in non-small cell lung cancer (NSCLC) have largely remained unclear.

Material and methods: In this study, we compared the PTTG3P expression levels between lung cancer and normal lung samples by analyzing 5 public datasets (GSE18842, GSE19804, GSE27262, GSE30219, and GSE19188). Next, pentose phosphate pathway and co-expression networks were constructed to identify key targets of IncRNA PTTG3P. Furthermore, gene ontology (GO) and Kyoto Encyclopedia of Genes and Genomes (KEGG) pathway analysis were performed to explore the potential roles of IncRNA PTTG3P. Moreover, we constructed PTTG3P-mediated ceRNA networks in lung adenocarcinoma (LUAD) and lung squamous cell carcinoma (LUSC).

Results: In the present study, our analysis showed that PTTG3P expression was higher in high T stage LUAD and LUSC samples, as well as high $\mathrm{N}$ stage NSCLC tissues. Of note, we found that higher PTTG3P expression is correlated with shorter survival time in NSCLC patients by analyzing Kaplan-Meier plotter datasets. We found that PTTG3P was significantly associated with NSCLC cell proliferation regulation by affecting a series of cell cycle related biological processes.

Conclusions: Bioinformatics analysis showed that PTTG3P was associated with NSCLC cell proliferation. These results suggested that PTTG3P could serve as a new therapeutic and prognostic target for NSCLC.

Key words: biomarker, long non-coding RNA, lung cancer, protein-protein interaction analysis, co-expression networks.

\section{Introduction}

Lung cancer is one of the most common fatal malignant tumors around the world [1, 2]. Approximately $80 \%$ of lung cancers were diagnosed as non-small cell lung cancer (NSCLC) and about $20 \%$ of lung cancers were small cell lung cancer (SCLC) [3]. Recently, a series of methods, including surgical therapy, radiation therapy, chemotherapy, targeted

\author{
Corresponding author: \\ Chong-Jun Zhong \\ the Second Affiliated \\ Hospital of \\ Nantong University \\ 6 North Hai'er \\ Lane Road \\ 226001 Nantong, China \\ Phone: +86051385061286 \\ Fax: +86 51385061003 \\ E-mail: zhongcjnt@126.com
}


therapy and immunotherapy, were developed for lung cancer treatment [4-6]. However, the survival rate for lung cancer is still low. Currently, several genes were identified as potential biomarkers for lung cancer. For example, circular RNA F-circEA was detected in the plasma of NSCLC patients and could act as a "liquid biopsy" biomarker [7]. LINC00473 was also up-regulated and associated with worse prognosis of lung cancer [8]. However, it is urgently necessary to identify novel sensitive and specific biomarkers for NSCLC.

Long noncoding RNAs (IncRNAs) are a kind of non-protein-coding RNAs longer than 200 bps $[9,10]$. Previous studies indicated that IncRNAs played crucial roles in gene transcription, posttranscription and epigenetic regulation [11, 12]. Various reports showed that IncRNAs were widely differently expressed in human cancers, including lung cancer, liver cancer, prostate cancer, and breast cancer $[13,14]$. Up-regulation of the long non-coding RNA RMRP contributes to glioma progression and promotes glioma cell proliferation and invasion [15]. In addition, many IncRNAs, such as MALAT1, LET, HOTTIP, and XIST, were suggested to be associated with the diagnosis of NSCLC [16]. High IncRNA HULC expression is associated with poor prognosis and promotes tumor progression by regulating epithelial-mesenchymal transition in prostate cancer [17]. Recently, several reports have highlighted pseudogenes, a type of special IncRNAs, playing special roles in tumor progression. For instance, PDIA3P interacted with c-Myc to promote G6PD expression and increase the pentose phosphate pathway (PPP) flux [18]. Small ubiquitin-like modifier 1 pseudogene 3 acted as an oncogene in colon and breast cancer [19]. The expression profiles of Notch pathway members have a significant influence on disease-free survival (DFS) in renal carcinoma [20]. The pituitary tumor-transforming 3 pseudogene (PTTG3P) was up-regulated in gastric cancer (GC) and could enhance GC cell proliferation and metastasis [21]. However, the clinical value and potential roles of PTTG3P in NSCLC have remained unclear.

Here, we hypothesized that PTTG3P could serve as a biomarker for NSCLC. We analyzed PTTG3P expression in lung cancer and normal lung samples by using Gene Expression Omnibus (GEO) datasets. Kaplan-Meier plotter (KM plotter) was also used to evaluate whether PTTG3P was associated with lung cancer survival time. Co-expression analysis in combination with bioinformatics analysis was used to predict PTTG3P potential functions.

\section{Material and methods}

\section{Microarray data}

A series of microarray datasets, including GSE18842, GSE19804, GSE27262, GSE30219, and GSE19188, were downloaded from GEO (https://www.ncbi.nlm.nih.gov/geo/) [22]. GeneChip Human Genome U133 Plus 2.0 Array included 6,500 additional genes for analysis of over 47,000 transcripts. The probe ID for PTTG3P is 208511_at.

Furthermore, we analyzed PTTG3P expression in lung adenocarcinoma (LUAD) and lung squamous cell carcinoma (LUSC) by using TCGA datasets, which were download from cBioPortal (http://www.cbioportal.org/) [23]. The methods of RNA isolation, RNA sequencing and data normalization were described previously. All the patients were staged using the 2009 Tumor-Node-Metastasis (TNM) classification of the American Joint Committee on Cancer/International Union Against Cancer [24].

\section{Survival analysis}

In the present study, we used Kaplan-Meier plotter (KM plotter) (http://kmplot.com/analysis/ index.php? $p=$ service\&cancer=lung) [25]. To investigate the clinical significance of LncRNA PTTG3P in NSCLC, we analyzed whether PTTG3P expression was correlated with survival time in NSCLC patients. The Kaplan-Meier plotter datasets included a total of 2437 lung cancer samples with a mean a mean follow-up of 49 months. In this study, we selected median expression of PTTG3P in all samples as a cutoff to divide into PTTG3Phigh and PTTG3P-low groups.

\section{Construction of PTTG3P-mediated ceRNA networks}

In this study, we predicted PTTG3P targeting miRNAs and mRNAs by using Starbase datasets, based on CLIP-seq. Next, we applied co-expression analysis for PTTG3P in TCGA LUAD and LUSC datasets using cBioPortal online software. Finally, we constructed PTTG3P-related ceRNA networks based on positive related PTTG3P-target (Pearson score $\geq 0.2$ ) pairs. Cytoscape (Version 3.4.0, available online: http://www.cytoscape.org/) was applied for visualization of the co-expression networks.

\section{GO and KEGG pathway analysis}

In this study, we performed GO and KEGG pathway analysis using the freely available online DAVID system (https://david.ncifcrf.gov/) [26]. A $p$-value less than 0.05 was considered as significant.

\section{Statistical analysis}

Statistical comparisons between two groups were performed using the $t$-test or Mann-Whit- 
ney $U$-test according to the test condition. For more groups, one-way ANOVA followed by the Newman-Keuls post hoc test was used. A $p$-value $<0.05$ was considered statistically significant.

\section{Results}

\section{Long non-coding RNA PTTG3P was}

up-regulated in non-small cell lung cancer

In this study, we compared the PTTG3P expression levels between lung cancer and normal lung samples by analyzing 5 public datasets: GSE18842, GSE19804, GSE27262, GSE30219, and GSE19188. As shown in Figures 1 A-E, PTTG3P was significantly overexpressed in lung adenocarcinoma (LUAD) and lung squamous cell carcinoma (LUSC) cancer patients compared to normal lung samples.

In order to further validate our finding, we analyzed TCGA datasets, which were based on RNA- seq methods. The RNA-seq method could unravel previously inaccessible complexities in the transcriptome, such as allele-specific expression and novel isoforms, and could also be used to detect gene expression in human disease samples. As shown in Figures $1 \mathrm{~F}, \mathrm{G}$, our analysis showed that PTTG3P was markedly overexpressed in LUAD and LUSC samples.

\section{LncRNA PTTG3P is correlated with pathology stage in NSCLC}

In order to evaluate the correlation between IncRNA PTTG3P expression and NSCLC clinicopathological characteristics, we further analyzed TCGA LUAD and LUSC datasets based on TNM stage. Our analysis showed that PTTG3P expression was higher in stage IA, stage IB, stage IIA, stage IIB, stage III and stage IV LUAD and LUSC samples than that in normal samples (Figures $2 \mathrm{~A}, \mathrm{~B})$. Interestingly, we also observed that PTTG3P was overexpressed in stage III and IV LUAD com-
A

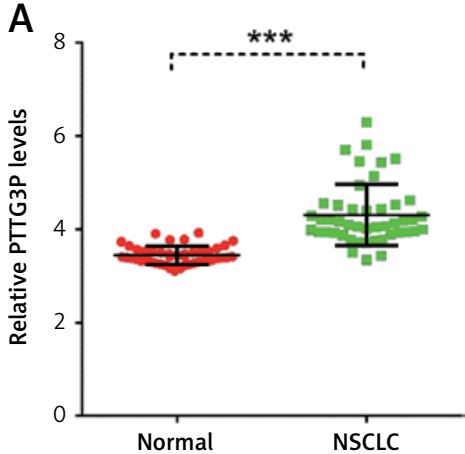

$D_{25}$

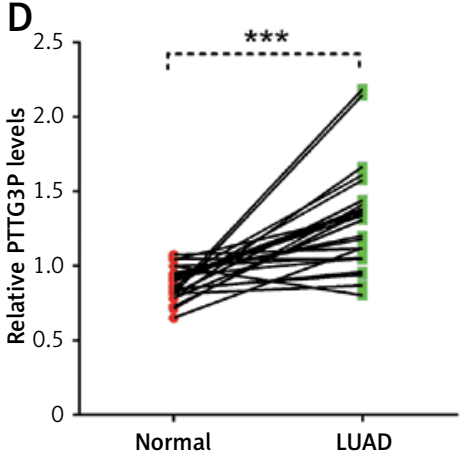

F

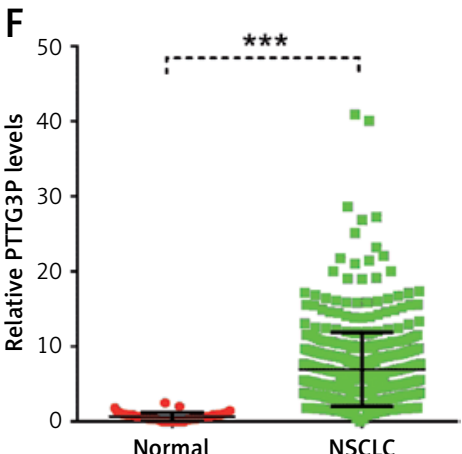

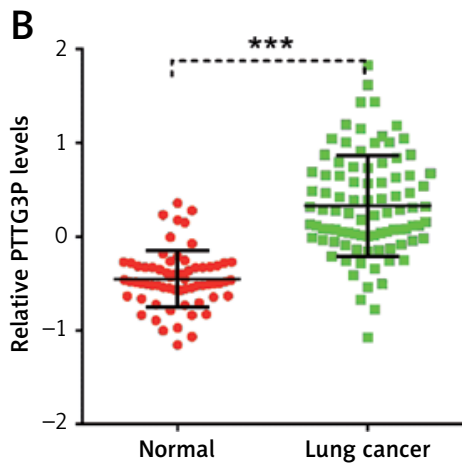
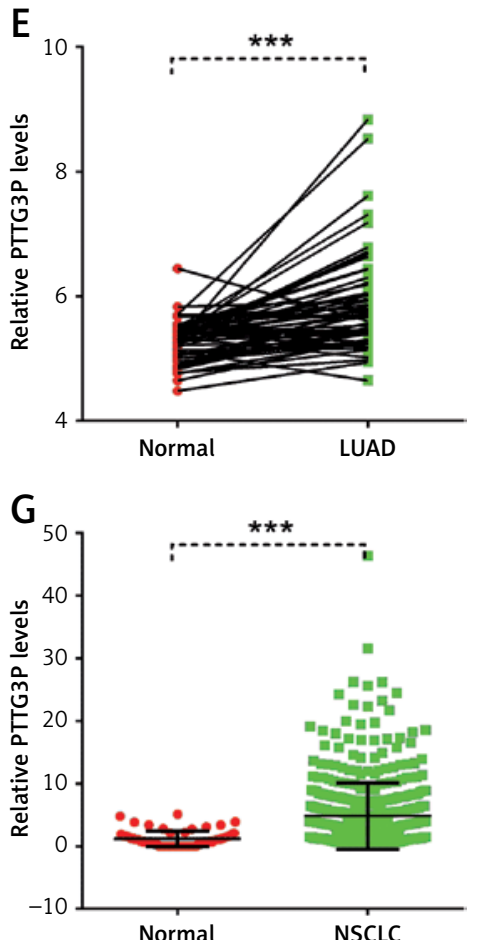

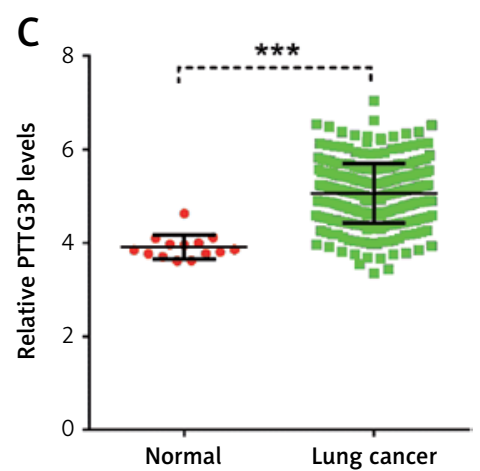

Normal

Figure 1. Long non-coding RNA PTTG3P was up-regulated in NSCLC. A-E - PTTG3P expression levels was up-regulated in GSE18842 (A), GSE19804 (E), GSE27262 (D), GSE30219 (C), and GSE19188 (B) datasets. F, G - PTTG3P expression levels was up-regulated in TCGA LUSC (F) and TCGA LUAD (G) datasets. Significance was defined as $p<0.05\left({ }^{*} p<0.05\right.$; $\left.{ }^{* *} p<0.01 ;{ }^{* *} p<0.001\right)$ 
A

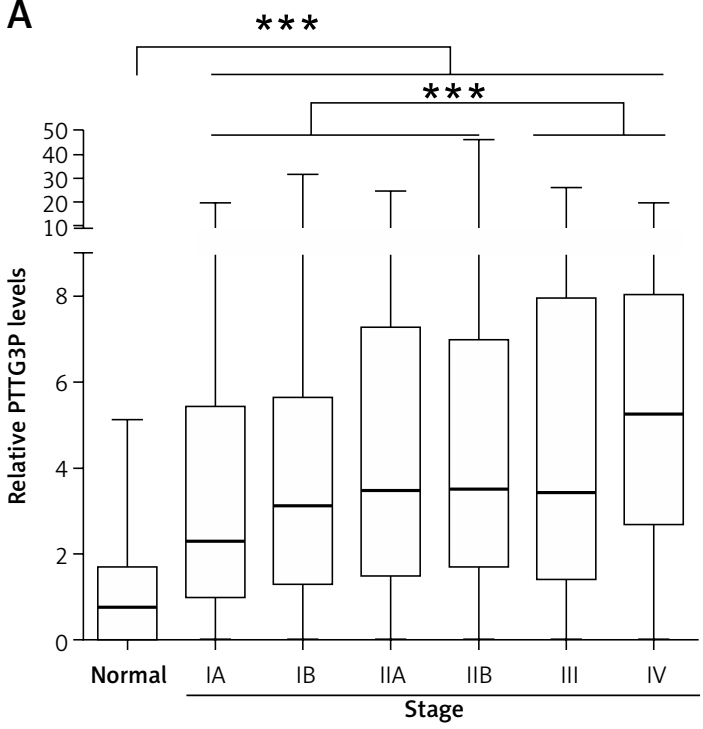

B

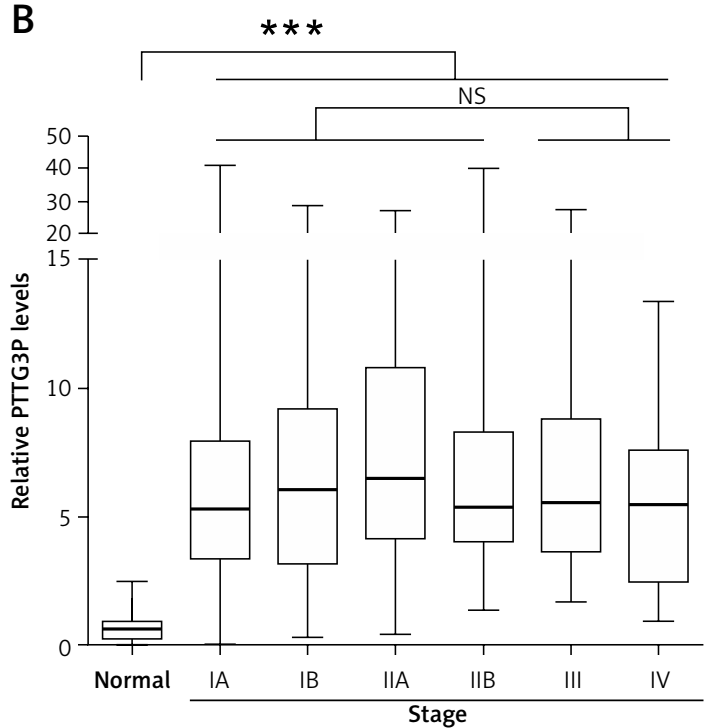

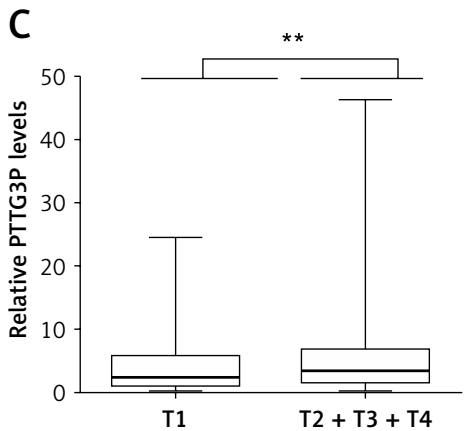
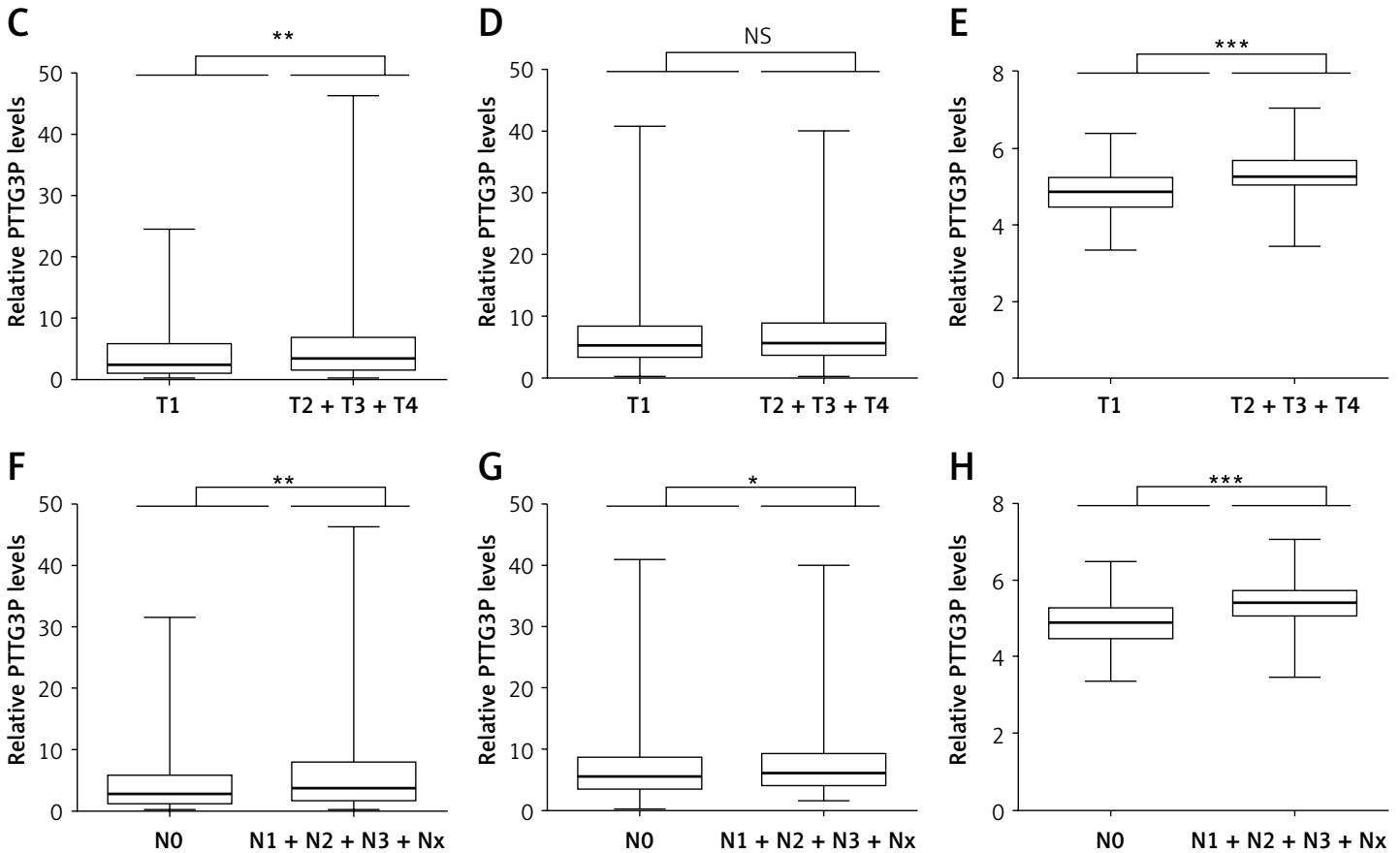

Figure 2. LnCRNA PTTG3P is correlated with pathological stage in NSCLC. The correlation between PTTG3P IncRNA expression and NSCLC clinicopathological characteristics by analyzing TCGA LUAD (A) and LUSC (B) datasets based on TNM stage. PTTG3P expression in NSCLC samples based on T staging and N staging by analyzing TCGA LUAD (C, F), LUSC (D, G) and GSE30219 (E, H) datasets

pared to stage I and stage II LUAD tissues (Figures $2 \mathrm{~A}, \mathrm{~B})$.

We then analyzed PTTG3P expression in NSCLC samples based on $\mathrm{T}$ staging and $\mathrm{N}$ staging. As shown in Figures $2 \mathrm{C}-\mathrm{H}$, we found that $\mathrm{T} 1$ stage lung cancer samples had lower PTTG3P expression compared to T2, T3 and T4 stage lung cancer tissues by analyzing TCGA LUAD, LUSC and GSE30219 datasets. We also observed the similar results that PTTG3P was highly expressed in high $\mathrm{N}$ stage lung cancer compared to NO stage lung cancer samples. Our analysis showed that LnCRNA PTTG3P was correlated with pathological stage in NSCLC.

\section{LncRNA PTTG3P is correlated with poor prognosis in NSCLC}

To further investigate the clinical significance of IncRNA PTTG3P in NSCLC, we analyzed whether PTTG3P expression is correlated with survival time in NSCLC patients. The Kaplan-Meier plotter datasets included a total of 2437 lung cancer samples with a mean a mean follow-up of 49 months. In this study, we selected median expression of PTTG3P in all samples as a cutoff to divide NSCLC patients into PTTG3P-high and PTTG3P-low groups. Our results showed that higher PTTG3P expression was 
A
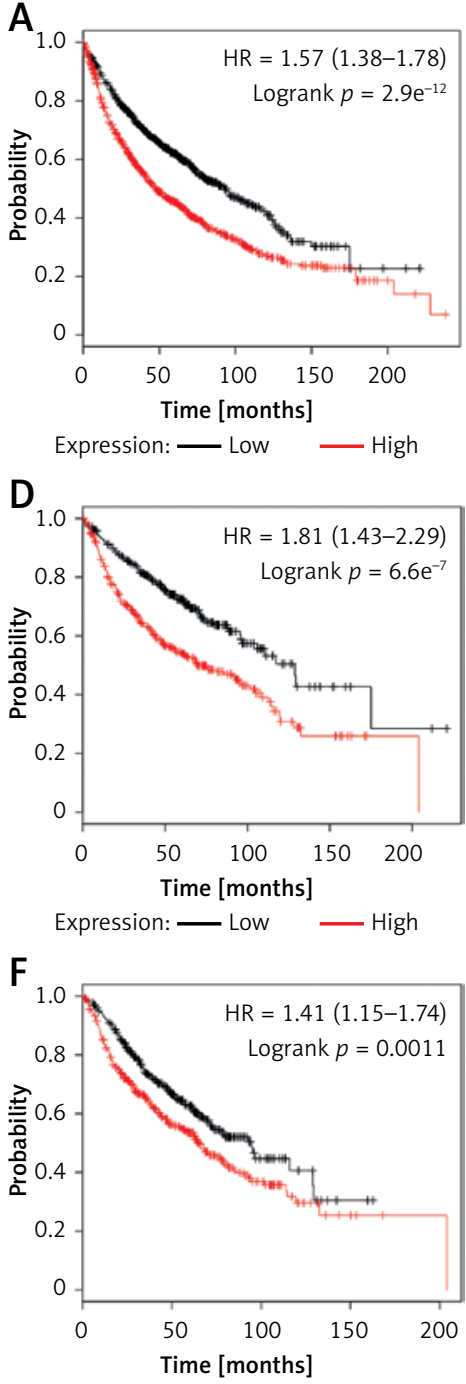

Expression: — Low High
B

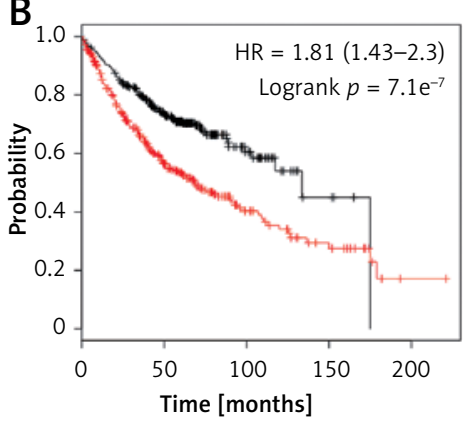

Expression: — Low High

$\mathrm{E}$

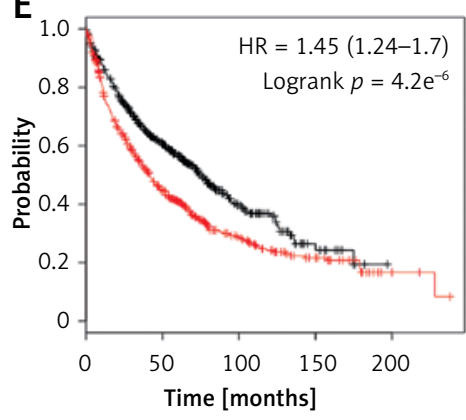

Expression: — Low High

G

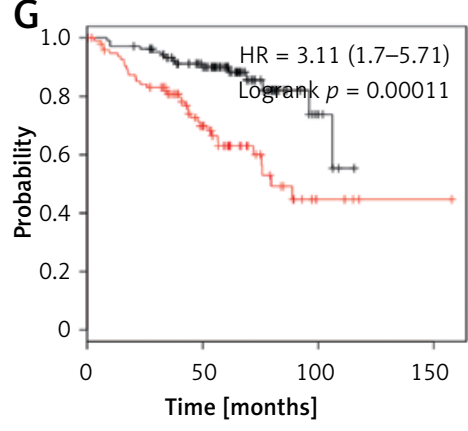

Expression: — Low High

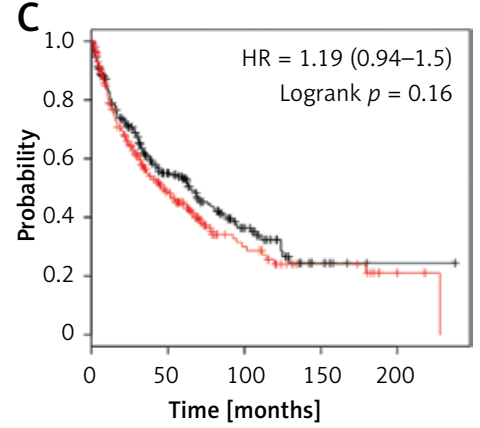

Expression: — Low High
Figure 3. LnCRNA PTTG3P is correlated with poor prognosis in NSCLC. The Kaplan-Meier plotter database analysis shows that the overall survival and disease-free survival times in the PTTG3P-high group were lower than those in the PTTG3P-IT1-low group in lung cancer (A), LUAD (B), LUSC (C), female lung cancer (D), male lung cancer (E), non-smoking lung cancer (F) and smoking cancer (G). Significance was defined as $p<0.05\left({ }^{*} p<0.05\right.$; $\left.{ }^{* *} p<0.01 ;{ }^{* * *} p<0.001\right)$ significantly correlated with shorter overall survival time in lung cancer. We then assessed the effect of PTTG3P on survival status of different types of lung cancer. The further analysis revealed that the overall survival time in the PTTG3P-high group was lower than that in the PTTG3P-low group in female NSCLC (Figure 3 D) and male NSCLC (Figure $3 \mathrm{E}$ ). Furthermore, we analyzed the correlation between PTTG3P expression and DFS time in lung cancer and observed a similar result (Figures 3 A-C, F, G). Our analysis strongly suggested that PTTG3P could act as a biomarker.

\section{LncRNA PTTG3P played different roles in different stages of NSCLC}

Considering that different histopathological subtypes had a different molecular disturbance, here, we explore the roles of PTTG3P in different stages of NSCLC. We performed co-expression analysis of PTTG3P in T1 stage and T2 + T3 + T4 stage LUAD. The PTTG3P-mRNAs with absolute
Pearson score values $\geq 0.2$ were considered as reliable regulators (Figure $4 \mathrm{~A}$ ).

We next performed KEGG pathway analysis of PTTG3P in T1 stage and T2 + T3 + T4 stage LUAD using Cytoscape's ClueGo plug-in. In KEGG pathway analysis PTTG3P was involved in regulating the cell cycle, p53 signaling, homologous recombination, DNA replication, spliceosome and mismatch repair in T1 stage LUAD. Our analysis showed that PTTG3P was involved in regulating DNA replication, one carbon pool by folate, pyrimidine metabolism, RNA transport, RNA degradation, the spliceosome, the 53 signaling pathway and the cell cycle in T2 + T3 + T4 stage LUAD (Figure $4 \mathrm{~B}$ ).

\section{Construction of PTTG3P-related ceRNA networks for NSCLC}

Previous studies had suggested that pseudogenes, a special type of IncRNAs, could serve as competing endogenous RNAs in cancer 
A

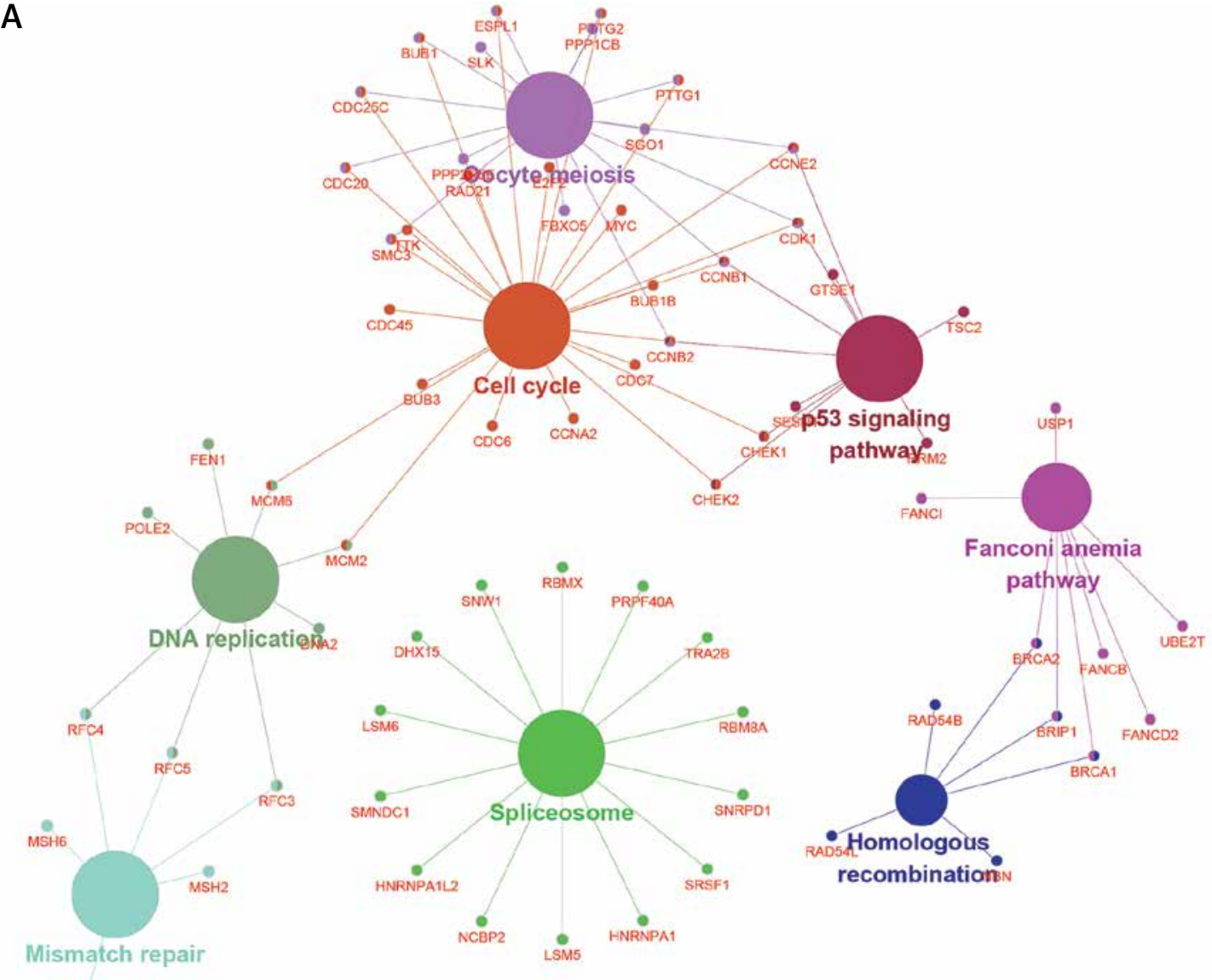

Exo!

B

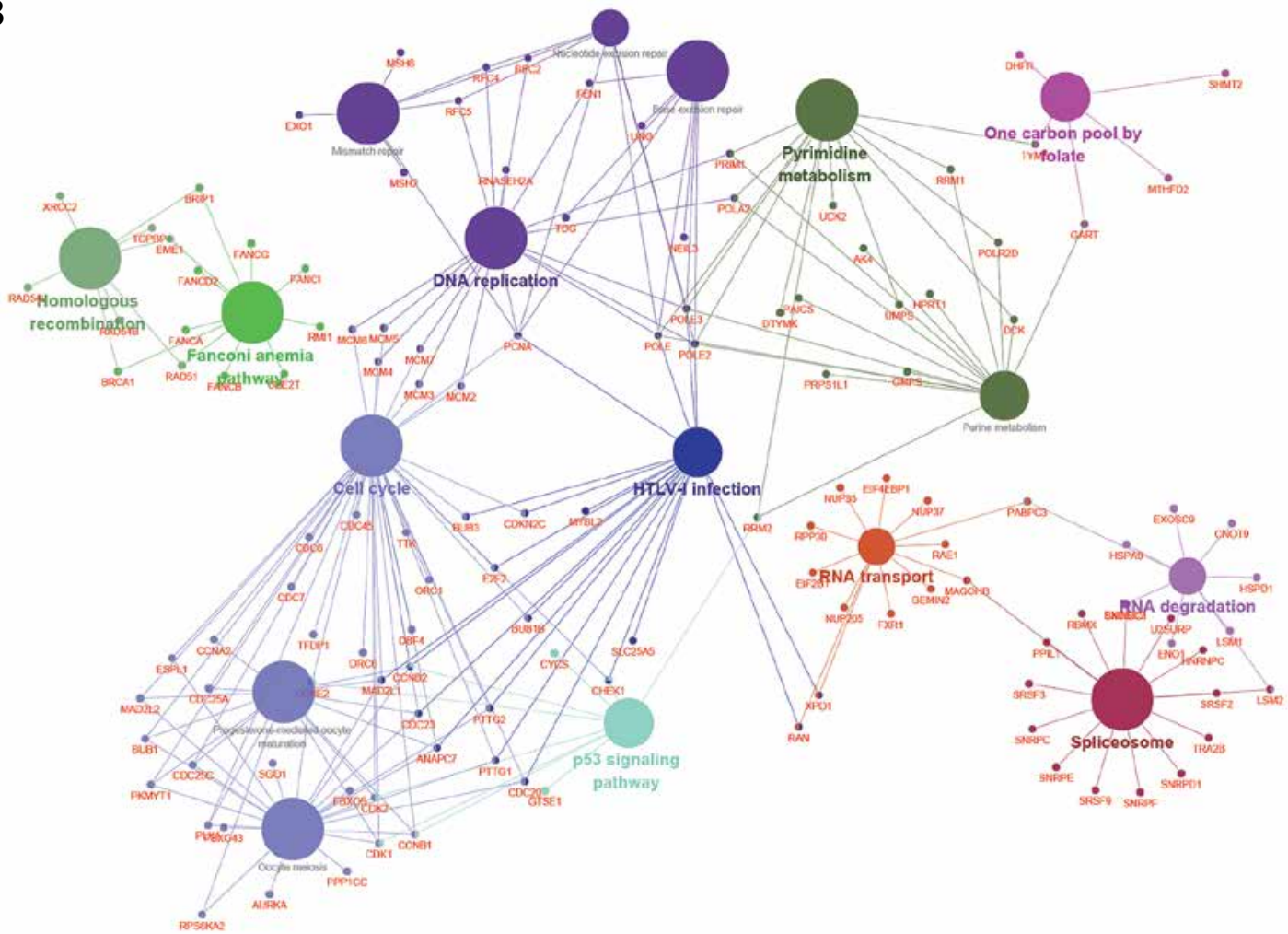

Figure 4. LncRNA PTTG3P played different roles in different stages of NSCLC. A - The co-expression analysis of PTTG3P in T1 stage and T2 + T3 + T4 stage LUAD. B - The KEGG pathway analysis of PTTG3P in T1 stage and T2 + T3 + T4 stage LUAD 
cells. Here, we also constructed PTTG3P-related ceRNA networks in NSCLC. We first predicted PTTG3P targeting miRNAs and mRNAs using Starbase datasets, based on CLIP-seq. Next, we applied co-expression analysis for PTTG3P in TCGA LUAD and LUSC datasets by using cBioPortal online software. Finally, we constructed PTTG3P-related ceRNA networks based on positive related PTTG3P-target (Pearson score $\geq 0.2$ ) pairs.

As shown in Figures $5 \mathrm{~A}$ and $\mathrm{B}$, two PTTG3P-related ceRNA networks were constructed for LUAD and LUSC. A total of 10 miRNAs, 584 mRNAs and 1300 edges were included in LUAD special ceRNA network, while 10 miRNA, 286 mRNAs and 582 edges were included in the LUSC special ceRNA network. Ten miRNAs (hsa-miR-129-5p, hsa-miR-3167, hsa-miR-376c-3p, hsa-miR-132-3p, hsa-miR-212-3p, hsa-miR-383-5p, hsa-miR-8765p, hsa-miR-873-5p, hsa-miR-421, hsa-miR-505$3 p)$ were identified as key regulators in PTTG3P-related ceRNA networks.

\section{Enrichment analysis of PTTG3P in NSCLC}

Our study showed that PTTG3P could serve as a biomarker for NSCLC, but the molecular functions of PTTG3P in NSCLC remained largely unclear. Here we performed enrichment analysis for PTTG3P based on ceRNA networks analysis. Our results showed that PTTG3P was involved in regulating cell division, DNA replication initiation, chromosome segregation, mitotic chromosome condensation, mitotic sister chromatid segregation, the cell cycle, double-strand break repair via homologous recombination, the response to ionizing radiation, positive regulation of isotype switching, positive regulation of telomere maintenance via telomerase, mRNA splicing, via spliceosome, protein folding, and the response to UV in LUAD (Figure 6 C). We also observed that PTTG3P was associated with cell division, mitotic nuclear division, sister chromatid cohesion, mRNA splicing via the spliceosome, mitotic sister chromatid segregation, the anaphase-promoting complex-dependent catabolic process, termination of RNA polymerase II transcription, mitotic spindle assembly checkpoint, negative regulation of ubiquitin-protein ligase activity involved in the mitotic cell cycle, positive regulation of ubiquitin-protein ligase activity involved in regulation of mitotic cell cycle transition, mRNA export from the nucleus, DNA replication, and translation in LUSC (Figure $6 \mathrm{~A}$ ).

KEGG pathway analysis revealed that PTTG3P was mainly enriched in the spliceosome, the cell cycle, RNA transport, the Fanconi anemia pathway, DNA replication, pyrimidine metabolism, base excision repair, homologous recombination, oocyte meiosis, and purine metabolism in LUAD (Figure $6 \mathrm{~B}$ ). In LUSC, we found that PTTG3P was enriched in the spliceosome, oxidative phosphorylation, the cell cycle, non-alcoholic fatty liver disease (NAFLD), RNA degradation, and progesterone-mediated oocyte maturation (Figure $6 \mathrm{D}$ ).

These results strongly suggested that PTTG3P plays important roles in regulating NSCLC cell proliferation by affecting cell cycle related biological processes.

\section{Discussion}

Non-small cell lung cancer (NSCLC) constitutes $80 \%$ of lung cancer cases. The 5 -year survival rate of NSCLC was as low as about 15\% [27]. However, the mechanisms underlying NSCLC progression remained unclear and novel sensitive and specific biomarkers for NSCLC were still lacking. Recently, several genes were identified as potential biomarkers for lung cancer. For example, MEG3 was down-regulated in lung cancer samples compared to normal tissues. Higher MEG3 expression levels were correlated with longer survival time in NSCLC [28]. IGF2AS was also reported to be suppressed in NSCLC samples [29]. In the present study, we evaluated the correlation between PTTG3P expression and clinical implications in NSCLC based on public datasets. Our results strongly suggested that PTTG3P could serve as a biomarker for NSCLC.

PTTG3P is a novel pseudogene, which has never been reported in lung cancer before. A previous study demonstrated that PTTG3P was up-regulated in gastric cancer (GC) and could enhance GC cell proliferation and metastasis, suggesting that PTTG3P serve as an oncogene in human cancer [21]. In this study, we compared the PTTG3P expression levels between lung cancer and normal lung samples and found that PTTG3P was significantly up-regulated in NSCLC samples by analyzing 5 public datasets: GSE18842, GSE19804, GSE27262, GSE30219, and GSE19188. Furthermore, we found that PTTG3P was correlated with pathological stage in NSCLC. Our analysis showed that PTTG3P expression was higher in high T stage LUAD and LUSC samples, as well as high N stage NSCLC tissues. Of note, we found that higher PTTG3P expression was correlated with shorter survival time in NSCLC patients by analyzing Kaplan-Meier plotter datasets. Moreover, we explored the roles of PTTG3P in different stages of LUAD. We found that PTTG3P was involved in regulating the cell cycle, p53 signaling, homologous recombination, DNA replication, the spliceosome and mismatch repair in T1 stage LUAD. Meanwhile, PTTG3P was involved in regulating DNA replication, one carbon pool by folate, pyrimidine metabolism, RNA transport, RNA degradation, the spliceosome, the 53 signaling pathway and the cell cycle in $\mathrm{T} 2+\mathrm{T} 3+\mathrm{T} 4$ LUAD samples. 


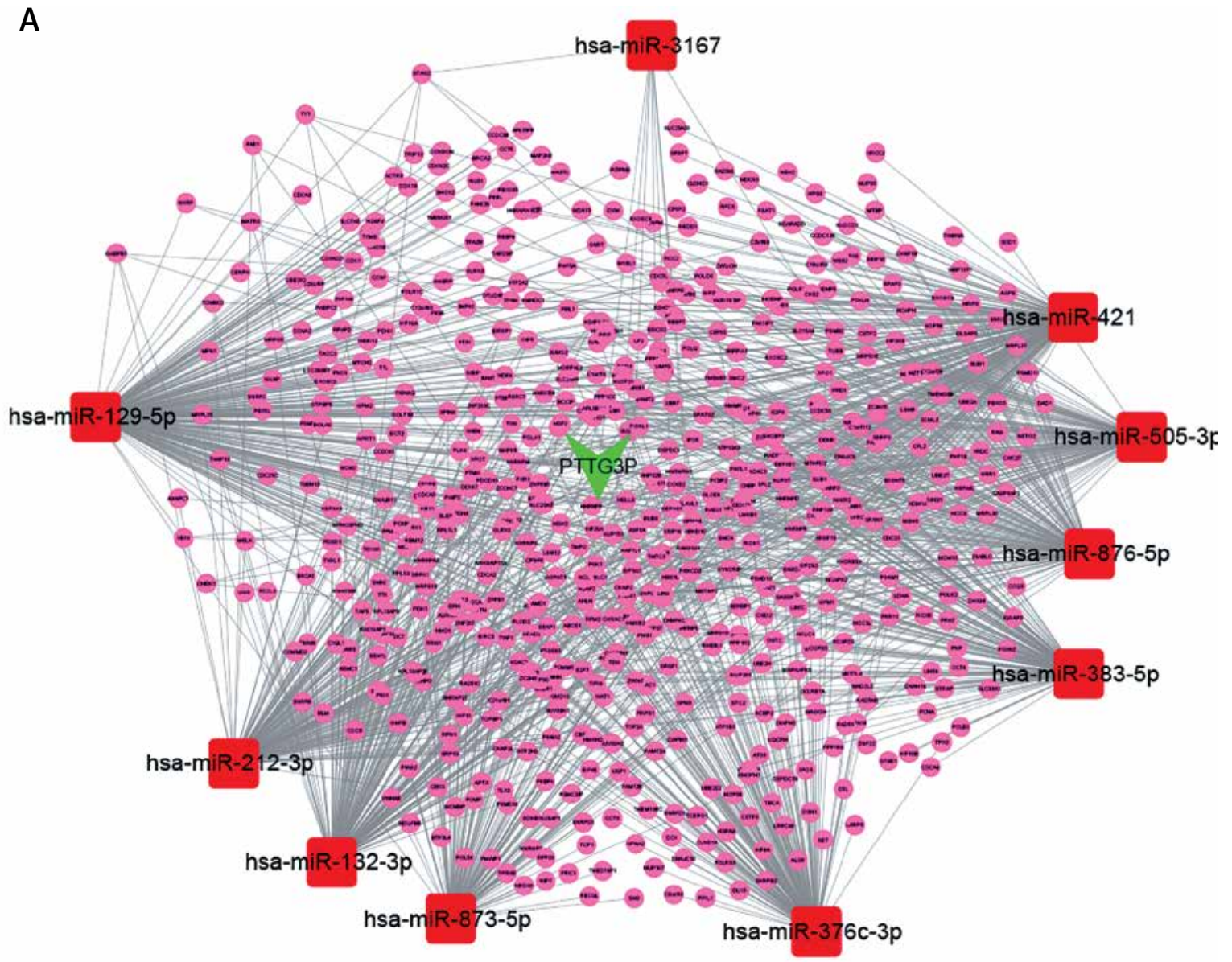

B

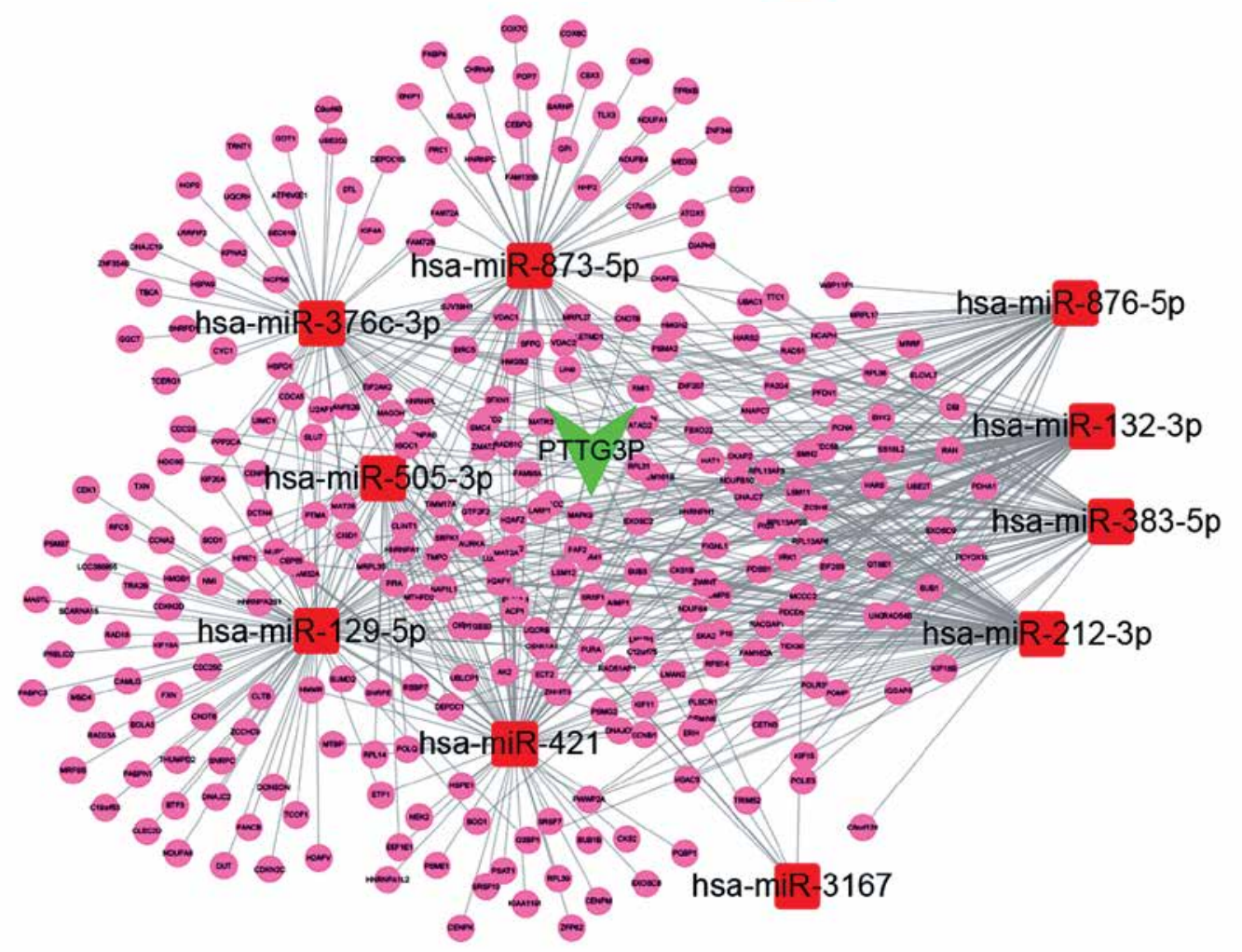

Figure 5. Construction of PTTG3P-related ceRNA networks for NSCLC. A - PTTG3P-related ceRNA networks were constructed in LUAD, 10 miRNAs, 584 mRNAs and 1300 edges were included in the network. B - PTTG3P-related ceRNA networks were constructed in LUSC, 10 miRNA, 286 mRNAs and 582 edges were included in the network 
A

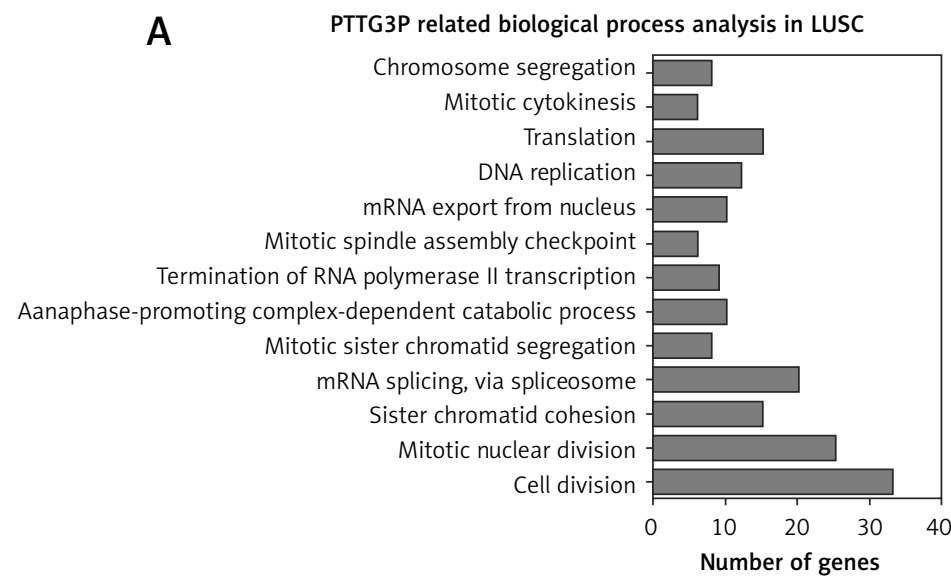

C

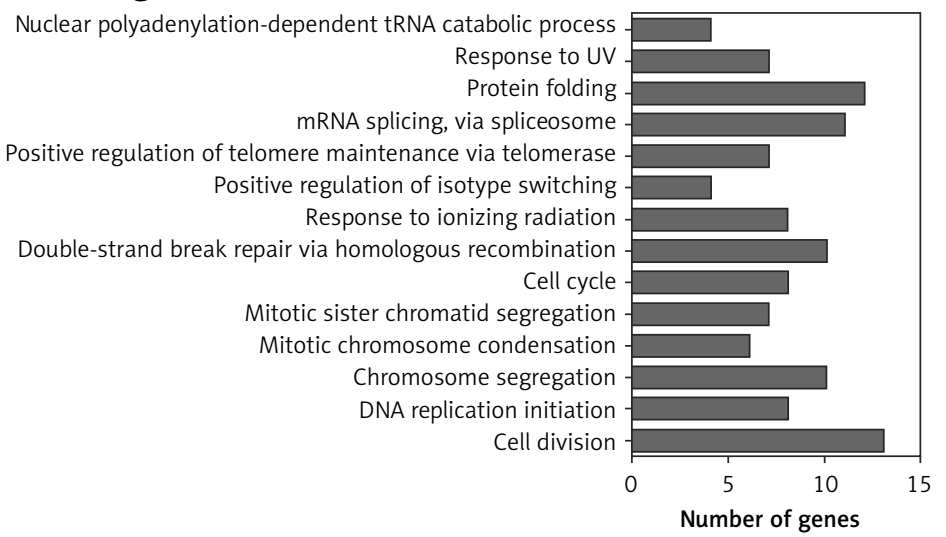

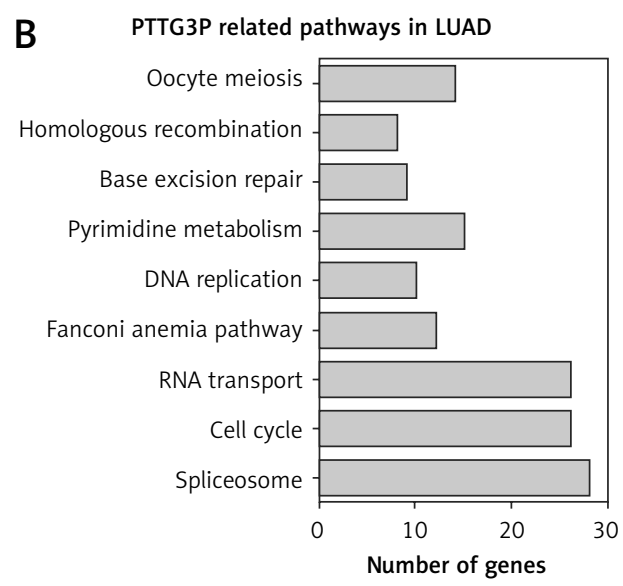

D

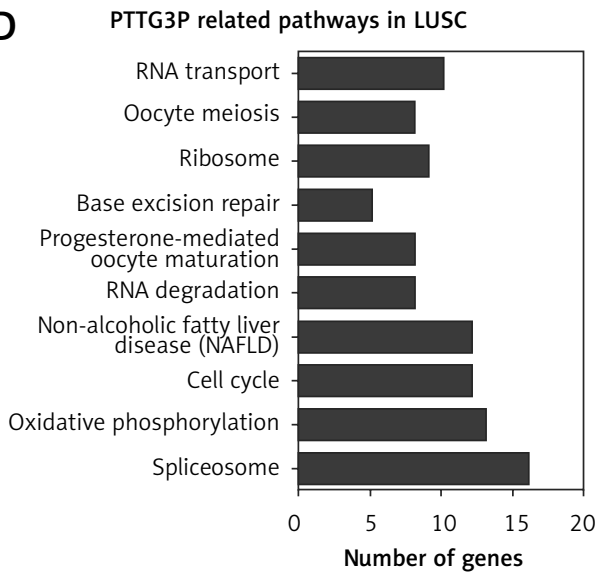

Figure 6. Enrichment analysis of PTTG3P in NSCLC. GO analysis shows PTTG3P related biological process analysis in LUSC (A) and LUAD (C). KEGG pathway analysis reveals PTTG3P-related pathways in LUSC (B) and LUAD (D)

Pseudogenes are a type of fossil relatives of protein-coding genes, which were thought of as "junk genes". However, emerging studies have shown that pseudogenes also play important roles in cancer progression by regulating cell proliferation, apoptosis and metastasis. For example, Poliseno and Pandolfi [30] found that PTENP1 could serve as a ceRNA to promote PTEN expression by sponging a series of miRNAs, including miR21, miR-17, miR-19 and miR-214. Ma et al. reported that a pseudogene DUXAP8 promoted gastric cancer cell progression by epigenetically silencing PLEKHO1 expression [31]. Another pseudogene, DUXAP10, was also found to promote colorectal cancer cell proliferation through epigenetically silencing of p21 and PTEN [32]. In this study, we for the first time explored the potential mechanism of PTTG3P regulating NSCLC progression by constructing mediated ceRNA networks in LUAD and LUSC. Ten miRNAs (hsa-miR-129-5p, hsamiR-3167, hsa-miR-376c-3p, hsa-miR-132-3p, hsamiR-212-3p, hsa-miR-383-5p, hsa-miR-876-5p, hsa-miR-873-5p, hsa-miR-421, hsa-miR-505-3p) were identified as key regulators in PTTG3P-related ceRNA networks. Furthermore, we performed bioinformatics analysis of PTTG3P based on ceRNA networks analysis. We found that PTTG3P was significantly associated with NSCLC cell proliferation regulation by affecting a series of cell cycle related biological processes, including ell division, DNA replication initiation, chromosome segregation, mitotic chromosome condensation, mitotic sister chromatid segregation, and the cell cycle.

In conclusion, our study showed for the first time that PTTG3P could serve as a biomarker of NSCLC. We observed that PTTG3P was significantly up-regulated in LUAD and LUSC samples by analyzing public datasets. We also found that PTTG3P expression was higher in high T stage and $\mathrm{N}$ stage NSCLC samples. Overexpression of PTTG3P was significantly correlated with shorter overall and disease-free survival time in lung cancer. Moreover, we constructed PTTG3P-mediated ceRNA networks in LUAD and LUSC. Bioinformatics analysis showed that PTTG3P was associated with NSCLC cell proliferation. These results must be confirmed in a prospective clinical trial as a new therapeutic target of NSCLC.

\section{Acknowledgments}

Hai-Tao Huang and Yi-Ming Xu contributed to this work equally. 
This work was supported by the project of Jiangsu Province Adolescent Medical Key Talent (project number: QNRC2016405)

\section{Conflict of interest}

The authors declare no conflict of interest.

\section{References}

1. Ding L, Getz G, Wheeler DA, et al. Somatic mutations affect key pathways in lung adenocarcinoma. Nature 2008; 455: 1069-75.

2. Chen W, Zheng R, Baade PD, et al. Cancer statistics in China, 2015. CA Cancer J Clin 2016; 66: 115-32.

3. Wistuba II, Gazdar AF, Minna JD. Molecular genetics of small cell lung carcinoma. Semin Oncol 2001; 28: 3-13.

4. Dillman RO, Seagren SL, Propert KJ, et al. A randomized trial of induction chemotherapy plus high-dose radiation versus radiation alone in stage III non-small-cell lung cancer. N Engl J Med 1990; 323: 940-5.

5. Shields T. Surgical therapy for carcinoma of the lung. Clin Chest Med 1993; 14: 121-47.

6. Morse MA, Garst J, Osada T, et al. A phase I study of dexosome immunotherapy in patients with advanced non-small cell lung cancer. J Transl Med 2005; 3: 9.

7. Tan S, Gou Q, Pu W, et al. Circular RNA F-circEA produced from EML4-ALK fusion gene as a novel liquid biopsy biomarker for non-small cell lung cancer. Cell Res 2018; 28: 693-5.

8. Chen Z, Li JL, Lin S, et al. CAMP/CREB-regulated LINC00473 marks LKB1-inactivated lung cancer and mediates tumor growth. J Clin Investig 2016; 126: 2267-79.

9. Zamore PD, Haley B. Ribo-gnome: the big world of small RNAs. Science 2005; 309: 1519-24.

10. Kornienko AE, Guenzl PM, Barlow DP, Pauler FM. Gene regulation by the act of long non-coding RNA transcription. BMC Biol 2013; 11: 59.

11. Wan X, Huang W, Yang S, et al. Identification of androgen-responsive IncRNAs as diagnostic and prognostic markers for prostate cancer. Oncotarget 2016; 7 : 60503-18.

12. Shi $X$, Sun M, Liu H, Yao Y, Song Y. Long non-coding RNAs: a new frontier in the study of human diseases. Cancer Lett 2013; 339: 159-66.

13. Zhang H, Chen Z, Wang X, Huang Z, He Z, Chen Y. Long non-coding RNA: a new player in cancer. J Hematol Oncol 2013; 6: 37.

14. Hauptman N, Glavač D. Long non-coding RNA in cancer. Int J Mol Sci 2013; 14: 4655-69.

15. Feng W, Li L, Xu X, Jiao Y, Du WJ. Up-regulation of the long non-coding RNA RMRP contributes to glioma progression and promotes glioma cell proliferation and invasion. Arch Med Sci 2017; 13: 1315-21.

16. Zhan Y, Zang H, Feng J, Lu J, Chen L, Fan S. Long non-coding RNAs associated with non-small cell lung cancer Oncotarget 2017; 8: 69174-84.

17. Zheng P, Li H, Xu P, et al. High IncRNA HULC expression is associated with poor prognosis and promotes tumor progression by regulating epithelial-mesenchymal transition in prostate cancer. Arch Med Sci 2018; 14: 679-86.

18. Yang $\mathrm{X}$, Ye $\mathrm{H}$, He $\mathrm{M}$, et al. LncRNA PDIA3P interacts with $c-M y c$ to regulate cell proliferation via induction of pentose phosphate pathway in multiple myeloma. Biochem Biophys Res Commun 2018; 498: 207-13.
19. Mei D, Song H, Wang K, et al. Up-regulation of SUMO1 pseudogene 3 (SUMO1P3) in gastric cancer and its clinical association. Med Oncol 2013; 30: 709.

20. Jedroszka D, Orzechowska M, Bednarek AK. Predictive values of Notch signalling in renal carcinoma. Arch Med Sci 2017; 13: 1249-54.

21. Weng W, Ni S, Wang Y, et al. PTTG3P promotes gastric tumour cell proliferation and invasion and is an indicator of poor prognosis. J Cell Mol Med 2017; 21: 3360-71.

22. Edgar R, Domrachev M, Lash AE. Gene Expression Omnibus: NCBI gene expression and hybridization array data repository. Nucleic Acids Res 2002; 30: 207-10.

23. Gao J, Aksoy BA, Dogrusoz U, et al. Integrative analysis of complex cancer genomics and clinical profiles using the cBioPortal. Sci Signal 2013; 6: pl1.

24. Rami-Porta R, Ball D, Crowley J, et al. The IASLC Lung Cancer Staging Project: proposals for the revision of the $T$ descriptors in the forthcoming (seventh) edition of the TNM classification for lung cancer. J Thorac Oncol 2007; 2: 593-602.

25. Győrffy B, Surowiak P, Budczies J, Lánczky A. Online survival analysis software to assess the prognostic value of biomarkers using transcriptomic data in non-small-cell lung cancer. PLoS One 2013; 8: e82241.

26. Ray JP, Staron MM, Shyer JA, et al. The interleukin2-mTORc1 kinase axis defines the signaling, differentiation, and metabolism of T helper 1 and follicular B helper T cells. Immunity 2015; 43: 690-702.

27. Mountain CF. Revisions in the international system for staging lung cancer. Chest 1997; 111: 1710-7.

28. Lu KH, Li W, Liu XH, et al. Long non-coding RNA MEG3 inhibits NSCLC cells proliferation and induces apoptosis by affecting p53 expression. BMC Cancer 2013; 13: 461.

29. Zhang X, Zhang X, Hu R, Hao L. Prognostic implication and functional role of long noncoding RNA IGF2AS in human non-small cell lung cancer. J Cell Biochem 2017; doi: $10.1002 / j c b .26113$.

30. Poliseno L, Pandolfi PP. PTEN ceRNA networks in human cancer. Methods 2015; 77: 41-50.

31. Ma HW, Xie M, Sun M, et al. The pseudogene derived long noncoding RNA DUXAP8 promotes gastric cancer cell proliferation and migration via epigenetically silencing PLEKHO1 expression. Oncotarget 2017; 8: 52211-24.

32. Lian Y, Xu Y, Xiao C, et al. The pseudogene derived from long non-coding RNA DUXAP10 promotes colorectal cancer cell growth through epigenetically silencing of p21 and PTEN. Sci Rep 2017; 7: 7312. 